\title{
Minimally Invasive Knee Arthroplasty with the Subvastus Approach Allows Rapid Rehabilitation: a Prospective, Biomechanical and Observational Study
}

Tsung-Ching Lin, MD, MS ${ }^{1)}$, Hsing-Kuo Wang, PhD²), Jia-Wine Chen, PhD $^{3)}$, Cheng-Ming Chiu, MD ${ }^{1}$, Hsiu-Ling Chou, $\mathrm{BS}^{4}$, Chin-Hung Chang, MD, $\mathrm{PhD}^{5-7}$ * $^{*}$

1) Department of Physical Medicine and Rehabilitation, Far Eastern Memorial Hospital

2) Sports Physiotherapy Laboratory, School and Graduate Institute of Physical Therapy, College of Medicine, National Taiwan University

3) Department of Mechanical Engineering, Oriental Institute of Technology

4) Department of Nursing, Taipei Veterans General Hospital

5) Division of Orthopedics, Department of Surgery, Far Eastern Memorial Hospital

6) Graduate School of Biotechnology and Bioengineering, Yuan Ze University

7) Department of Orthopaedic Surgery, National Taiwan University Hospital: No.21, Sec. 2, Nanya S. Rd., Banciao Dist., New Taipei City 220, Taiwan (R.O.C.). TEL: +886 2-89667000-2888,

FAX: $+8862-89665567$

\begin{abstract}
Purpose] To study the recovery of patients treated with minimally invasive total knee arthroplasty (TKA) performed via the subvastus approach, and to develop an optimal rehabilitation program for these patients. [Methods] Twenty-two patients (17 females and 5 males; mean age 69.2 years), who received unilateral minimally invasive TKA for osteoarthritis, underwent isometric and isokinetic muscle testing and completed a quality of life questionnaire, the Western Ontario and McMaster Universities Osteoarthritis Index (WOMAC), before and after surgery. Muscle strength and ultrasound tests were repeated 1, 2, 6, and 12 months after surgery. [Results] Strength and range of motion were initially lower in the operated knees but demonstrated no significant difference from the healthy knees after 12 months. Sonographically, joint effusion was greater in the osteoarthritic knees than in the healthy knees at baseline, but no significant difference was observed after 12 months. The mean WOMAC pain, stiffness and function scores all decreased from baseline to 6 months, and then slightly increased at 12 months, but only the function score showed a significant difference compared to baseline. [Conclusions] One year after minimally invasive TKA using a subvastus approach, patients had a good overall prognosis, with prompt functional recovery.

Key words: Arthroplasty, Knee prosthesis, Osteoarthritis
\end{abstract}

(This article was submitted Nov. 1, 2012, and was accepted Dec. 25, 2012)

\section{INTRODUCTION}

Osteoarthritis is the most common cause of long-term disability in most persons over the age of 65 , and the knee is the most frequent target ${ }^{1,2)}$. Eighty percent of patients with osteoarthritis have limitation of movement, and $25 \%$ have difficulty with activities of daily living. The annual economic cost arising from osteoarthritis in the US has been estimated to be $\$ 60$ billion $^{3)}$.

Total knee arthroplasty (TKA) is a major operation that is accompanied by significant postoperative pain during the aggressive rehabilitation that is essential for a good outcome and restoration of mobility. Most patients need assistive devices for at least 6 weeks or longer, until the femoral muscle incision heals. They also face several months of fre-

*To whom correspondence should be addressed.

E-mail: orthocch@mail.femh.org.tw quent sessions of physical therapy and continuing need for narcotic analgesics.

The development of minimally invasive TKA over the past 10 years has provided many advantages over traditional TKA. Patients who undergo the minimal procedure usually have less postoperative pain, a shorter stay in the hospital, and faster return to control of the quadriceps muscle, helping shorten the period of dependence on assistive devices. The shorter incision used with minimally invasive TKA-usually 4 to 5 inches (depending on the individual patient) versus 6 to 9 inches with the standard approachalso spares the quadriceps tendon. While standard TKA involves lateral eversion of the patella and dislocation of the tibiofemoral joint, with the minimally invasive approach, the patella is displaced without eversion, and joint dislocation can usually be avoided or minimized.

Despite all these advantages, minimally invasive approaches, including parapatellar, midvastus, and subvastus 
approaches, are not all alike. Theoretically, the subvastus approach should provide the best recovery with early rehabilitation because it causes the least damage to the quadriceps during surgery. While arthroplasty provides a mechanical solution to a biological problem, it does not modify the underlying disease. Much remains to be learned about the long-term effects of TKA on functional impairment and physical limitations. This also has ramifications for rehabilitation programs.

With all of this in mind, we evaluated the recovery of a group of patients who underwent minimally invasive TKA with the subvastus approach by comparing postoperative strength, anatomic changes, and function of the surgically treated knee compared with the patient's healthy untreated knee. Our long-term goal is to develop an optimal rehabilitation program for patients undergoing minimally invasive TKA.

\section{SUBJECTS AND METHODS}

\section{Subjects}

Twenty-two patients with osteoarthritis who were scheduled to undergo minimally invasive TKA performed by the same orthopedic surgeon at a medical center in New Taipei City were enrolled in this study. Inclusion criteria included: unilateral osteoarthritis; no history of minimally invasive TKA; and a preoperative range of motion $>10-90$ degrees. All patients provided their written informed consent (FEMH IRB No. 96053) to participation in this study. Patients were excluded from the study if they had any of the following: neural disorders causing cognitive dysfunction (i.e., scores less than 20 on the mini-mental status examination, or MMSE); diabetes, with a fasting glucose level $\geq$ $250 \mathrm{mg} / \mathrm{dL}$ plus ketosis, or fasting plasma glucose levels $\geq$ $300 \mathrm{mg} / \mathrm{dL}$; neural disorders due to diabetes or other diseases, causing dysfunction of proprioception or affecting lower extremity motor function; hypertension, diastolic pressure $>110 \mathrm{~mm} \mathrm{Hg}$ or systolic pressure $>180 \mathrm{~mm} \mathrm{Hg}$; congestive heart failure (New York Heart Association categories III and IV); hip or ankle disorders, or peripheral vascular disease; acute infection of the knee joint; or (8) incomplete soft tissue coverage. Although there was no control group per se, several strength studies of patients receiving TKA have used patients' healthy knees as a control ${ }^{4}$, and we followed this protocol.

\section{Methods}

All patients underwent biomechanical muscle strength tests and ultrasound measurements. All muscle strength tests were performed using an isokinetic dynamometer (Biodex System 3 Pro; Biodex Medical Systems, Inc., Shirley, NY). The dynamometer measured range of motion (ROM), maximal isometric muscle strength, peak isokinetic torque of the quadriceps and hamstrings, and the hamstring-to-quadrics ratio ( $\mathrm{H} / \mathrm{Q}$ ratio). The rate of force development was also obtained.

Patients also underwent isometric and isokinetic testing. During the tests the patients were seated with the hip flexed at 80 degrees and the knee flexed at 90 degrees. Then, the maximum stretch and contraction powers of the quadriceps and hamstrings muscle were measured. Thirty degrees and 60 degrees were preset as the measurement angles. For the isokinetic test, the patient was seated, with 80 degrees of hip flexion and 90 degrees of knee flexion, and maximum isokinetic contractions of the quadriceps muscle and hamstrings muscles were then performed. For statistical analysis, the maximal isometric muscle strength values were adjusted for patients' body weights. The angular velocities were 60 degrees/sec and 180 degrees/sec. ROM was preset to $10-80$ degrees.

The rate of force development (RFD) was calculated with continuous data, as the slope of the change in the initial isometric contraction of the time-force curve (the mean slope of delta force to delta time ( $\Delta$ force/ $\Delta$ time). The beginning of contraction was defined as the moment of adding 3.5 newton meters compared to the rest period, which is equal to $2 \%$ of the maximum torque ${ }^{5}$.

Ultrasound measurements were made with a 5- to 12$\mathrm{MHz}$ linear array (Philips HDI 5000; SonoCT, Bothwell, WA). The thicknesses of the sagittal and transverse crosssections of the quadriceps were measured with the patient supine. With the patient supine, and both knees extended, a probe was placed horizontally at the center of the anterior thigh (at the center of the line between the greater trochanter and the upper margin of the patella). Then the thickness and cross-sectional area of the rectus femoris (RF), vastus lateralis (VL), and vastus intermedius (VI), sartorius (SA) and vastus medialis (VM) were measured. In addition, measurements were made of the suprapatellar pouch effusion within the knee joint, the thickness of the intercondylar cartilage, and the thickness of the quadriceps and patellar tendon.

Patients also completed the Chinese version of the Western Ontario and McMaster Universities Osteoarthritis In$\operatorname{dex}(\text { WOMAC })^{6}$. The WOMAC, which is used worldwide to measure lower extremity function, consists of 24 items divided into 3 subscales: pain, stiffness, and physical function. Subsequently, all patients underwent preoperative conditioning, performing mobility exercises to help condition the muscles and ligaments for the postoperative rehabilitation protocol.

All 22 patients received minimally invasive TKA at our hospital. Using spinal or general anesthesia and with the patient supine and the knee slightly flexed, a straight midline incision was begun medial to the quadriceps tendon and $1 \mathrm{~cm}$ above the superior pole of the patella. The incision was extended distally to the level of the tibial tubercle, and a medial subvastus capsular incision was made. All patients received a Zimmer LPS-Flex Legacy ${ }^{\circledR}$ Fixed Bearing Knee implant (Zimmer, Warsaw, IN), which is specially designed to provide extensive flexion of the knee.

The posterior cruciate ligament was completely (the prosthesis has a posterior cruciate ligament substitution design). Soft tissue release was performed according to the preoperative deformity. After the distal femoral and proximal tibial cuts were made, a spreader was used to create a rectangular extension space, and the extension gap was measured. After resecting the tibia and sizing the femoral 
component (within $2 \mathrm{~mm}$ of the measured anatomy), an appropriately sized implant was inserted, and the patellar, femoral, and tibial components were fixed with cement fixation. The incision was closed with a "drop and dangle" test of the knee to predict the range of motion for the patient and a "no-thumb test" for patellar tracking. The knee was then positioned in full extension to continue closing the individual layers with hemovac drainage. Patient-controlled analgesia was used immediately after surgery, and all patients were placed in a continuous passive motion machine the same day of operation.

Minimally invasive TKA using the subvastus approach is a common procedure at our hospital, and a physician from the rehabilitation department designs a standard rehabilitation plan for each patient. One day after surgery, patients are asked to perform ankle-pumping exercises in bed for the initial stage of rehabilitation. Continuous passive motion (CPM) of the operated knee, with the ROM of the operated knee set at 0 to 40 degrees, is also provided. CPM is performed 3 times a day, and cryotherapy is applied after each session to prevent pain. CPM is then increased by 10 to 15 degrees each day, and a pillow is placed under the ankle to keep the knee in extension.

In addition, active movements of the primary joints in the lower limbs are introduced, with assistance, in order to prevent disuse atrophy and reduction of muscular strength. Two to three days after surgery, patients are encouraged to stand and walk. Seated knee flexion and extension are also encouraged to increase flexibility at the knee joint. The goal at discharge is for the patients to achieve at least 0-90 degrees ROM with the operated knee. After discharge, patients are required to return regularly for follow-up. Also, patients are instructed to continue with a home exercise plan to help restore muscular strength and improve function. All patients receive training for routine postsurgical self-care and walking, and are discharged from our hospitals, 5 or 6 days after surgery.

In the middle stage of rehabilitation, 1 to 3 weeks after surgery, the operated knees are iced, when necessary, and patients are asked to continue with lower limb strength training. Also, it is important that the ROM of the knee joint be steadily increased, so that knee flexion is $>105$ degrees and extension 0 degrees. At this stage, patients are expected to be able to change positions independently, such as sitting up from a lying position, and rising from the bed and moving around the room. Patients are also encouraged to train independently, practicing rising from chairs of various heights, and continuing with proper gait training and activities of daily living.

Three to 8 weeks after surgery, adjunctive hydrotherapy is applied to facilitate circulation. The lower limb exercises from the initial and mid-stages of rehabilitation continue, and the intensity of the exercises is increased by augmenting the number of repetitions or adding the proper amount of resistance or weights as tolerated. Patients continue to practice knee ROM and flexibility exercises. At this stage, the goal is to flex the operated knee more than 115 degrees. Patients also practice going up and down stairs, with a goal of evenly balancing their weight on both legs. They also use a stationary bike to increase lower limb and cardiovascular endurance. Proprioception and balance training are performed during the same visit, and patients prepare to return to their pre-surgery work and activities.

All patients are then followed for a year after surgery. Follow-up muscle strength tests and ultrasound measurements are repeated at 1, 2, 6, and 12 months after surgery.

Data are summarized as the median with inter-quartile range (IQR; range the 25 th and 75 th percentile). Due to the small sample size, nonparametric statistical methods were used. Changes in the osteoarthritic and healthy knees during the 12-month follow-up period were tested with the Friedman test, and the differences between two time points were tested with the Wilcoxon signed-rank test. Correlations between strength and functional parameters were assessed with the Pearson's correlation coefficient. $\mathrm{p}<0.05$ were considered to be statistically significant. Statistical analyses were performed using SPSS 15.0 statistics software (SPSS Inc., Chicago, IL).

\section{RESULTS}

A total of 22 patients with osteoarthritis who met the inclusion criteria were enrolled in this study: 17 females and 5 males, with a mean age of 69.2 years.

Before surgery, significantly lower ROM was observed in the knees with osteoarthritis than in the healthy knees (median: 100.0 vs. $120.0 ; \mathrm{p}<0.001$ ). Maximal isometric muscle strength of the hamstrings (IMH) at 30 degrees, and peak isokinetic strength and torque of the quadriceps (IKQ) at 180 degrees of the knees with osteoarthritis were significantly lower than their respective values in healthy knees (median: IMH 30: 21.0 vs. 29.2, respectively; $p=0.014$; median IKQ 180: 44.1 vs. 45.9 , respectively; $p=0.007$ ). The ultrasound parameters, transverse cross-section thickness of the vastus intermedius (VITR) osteoarthritic vs. healthy (1.12 vs. 1.33 , respectively; $\mathrm{p}=0.003)$, transverse crosssection thickness of the vastus lateralis (VLTR) (1.11 vs. 1.38 , respectively; $\mathrm{p}<0.001$ ), and transverse cross-section thickness of the vastus medialis (VMTR) ( 0.99 vs. 1.12, respectively; $p=0.002$ ) were significantly lower in the knees with osteoarthritis than in the healthy knees, and greater effusion was observed in the knees with osteoarthritis compared to the healthy knees ( 0.50 vs. 0.25 , respectively; $p$ $<0.001)$.

One month after surgery, ROM, RFD, isometric and isokinetic peak strengths of the operated knees were significantly lower than those of the healthy knees (Table 1). The ultrasound parameters, including VITR, VLTR, and muscle thickness on the longitudinal view of the vastus lateralis (VLSAG), were significantly lower for the operated knees than for the healthy knees, and higher effusion was observed in the operated knees than the healthy knees (Table 2).

The significant differences between the two knees in isometric and isokinetic peak strength before and 1 month after the operation indicate the possible impact on muscle strength of the severity of osteoarthritis and the operation itself. At baseline and 1 month post-operation, IMH of the operated knees, measured at 30 degrees, was significantly lower than that of the healthy knees. At 1 and 2 months 
Table 1. Patient characteristics at baseline and 1 month post-operation

\begin{tabular}{lcccc}
\hline & \multicolumn{2}{c}{ Baseline } & \multicolumn{2}{c}{1 month post-operation } \\
& Operated side & Healthy side & Operated side & Healthy side \\
\hline RFD50 & $0.122(0.030,0.207)$ & $0.118(0.097,0.176)$ & $0.101(0.045,0.134)^{*}$ & $0.113(0.062,0.173)$ \\
RFD100 & $0.078(0.041,0.136)$ & $0.074(0.060,0.125)$ & $0.065(0.026,0.085)^{*}$ & $0.074(0.037,0.132)$ \\
RFD150 & $0.060(0.033,0.151)$ & $0.061(0.044,0.115)$ & $0.050(0.022,0.071)^{*}$ & $0.054(0.026,0.121)$ \\
ROM & $100.0(90.0,116.3)^{*}$ & $120.0(115.8,130.0)$ & $84.0(77.0,104.0)^{*}$ & $116.5(104.0,126.3)$ \\
IMQ30 & $21.5(18.2,26.8)$ & $23.7(14.0,32.3)$ & $17.5(12.9,24.7)^{*}$ & $30.7(14.3,34.9)$ \\
IMQ60 & $45.1(28.8,62.5)$ & $50.4(32.4,57.8)$ & $34.9(20.2,45.9)^{*}$ & $60.4(31.9,85.9)$ \\
IMH30 & $21.0(11.3,36.9)^{*}$ & $29.2(16.3,47.0)$ & $19.5(8.3,28.3)^{*}$ & $26.6(11.1,35.0)$ \\
IMH60 & $22.9(11.8,34.3)$ & $22.8(14.7,38.1)$ & $24.0(6.9,31.0)^{*}$ & $25.4(15.1,39.3)$ \\
IKQ60 & $50.0(35.7,71.7)$ & $60.8(43.7,92.4)$ & $21.6(16.1,44.4)^{*}$ & $42.9(32.0,66.6)$ \\
IKQ180 & $44.1(26.9,51.5)^{*}$ & $45.9(41.0,59.7)$ & $34.9(24.9,47.6)^{*}$ & $52.5(37.7,68.0)$ \\
IKH60 & $35.6(25.3,53.2)$ & $35.9(21.7,60.8)$ & $36.5(25.8,44.8)^{*}$ & $64.8(46.0,95.2)$ \\
IKH180 & $44.1(30.7,51.3)$ & $43.9(32.7,61.4)$ & $30.2(24.9,39.6)^{*}$ & $46.4(39.6,66.9)$ \\
\hline
\end{tabular}

* Significant difference between the operated and healthy knees.

Key: RFD: rate of force development, IMQ: maximal isometric muscle strength of the quadriceps, IMH: maximal isometric muscle strength of the hamstrings, IKQ: peak isokinetic torque of the quadriceps, IKH: peak isokinetic torque of hamstrings.

Table 2. Ultrasound measurements of the 22 patients

\begin{tabular}{lllccc}
\hline \multirow{2}{*}{ VITR } & Operated & $\begin{array}{c}\text { Pre-operation } \\
(\mathrm{n}=22)\end{array}$ & $\begin{array}{c}1 \text { month } \\
\text { post-operation } \\
(\mathrm{n}=20)\end{array}$ & $\begin{array}{c}2 \text { months } \\
\text { post-operation } \\
(\mathrm{n}=18)\end{array}$ & $\begin{array}{c}6 \text { months } \\
\text { post-operation } \\
(\mathrm{n}=16)\end{array}$ \\
& Healthy & $1.12(0.91,1.25)^{*}$ & $0.97(0.83,1.13)^{*}$ & $0.98(0.87,1.29)$ & $1.14(0.86,1.42)$ \\
VLTR & Operated & $1.11(0.91,1.37)^{*}$ & $1.01(0.81,1.27)^{*}$ & $1.03(0.81,1.13)^{*}$ & $1.04(0.93,1.28)$ \\
& Healthy & $1.38(1.19,1.55)$ & $1.27(1.14,1.47)$ & $1.22(1.11,1.43)$ & $1.17(0.98,1.45)$ \\
VMTR & Operated & $0.99(0.75,1.11)^{*}$ & $1.12(0.93,1.26)$ & $0.95(0.77,1.24)$ & $0.89(0.77,1.00)$ \\
& Healthy & $1.12(0.92,1.43)$ & $1.00(0.89,1.07)$ & $0.96(0.81,1.17)$ & $0.94(0.77,1.09)$ \\
VLSAG & Operated & $1.16(0.92,1.48)$ & $1.03(0.83,1.14)^{*}$ & $0.93(0.81,1.16)^{*}$ & $1.11(0.93,1.32)$ \\
& Healthy & $1.24(1.16,1.44)$ & $1.22(1.09,1.40)$ & $1.31(1.12,1.53)$ & $1.16(0.98,1.39)$ \\
Effusion & Operated & $0.50(0.38,0.61)^{*}$ & $0.50(0.37,0.56)^{*}$ & $0.55(0.42,0.65)^{*}$ & $0.54(0.49,0.64)^{*}$ \\
& Healthy & $0.25(0.14,0.40)$ & $0.20(0.10,0.55)$ & $0.23(0.10,0.37)$ & $0.44(0.25,0.53)$ \\
\hline
\end{tabular}

* Significant difference between the operated and healthy knees.

post-operation, IMH measured at 60 degrees was significantly lower than that of the healthy knees. IMQ measured at 30 degrees was significantly lower than that of the healthy knees 1 month post-operation. IMQ measured at 60 degrees was significantly lower than that of the healthy knees 1 and 2 months post-surgery.

Both peak isokinetic strength and torque of the hamstrings (IKH) measured at 60 and 180 degree/sec on the operated side had decreased at 1 month post-operation, but then continuously increased up to the 12 -month point. IKH of the operated side measured at 60 and 180 degrees was significantly higher 12 months after surgery than at baseline (data not shown).

Both IKQ measured at 60 and 180 degrees on the operated side had decreased at 1 month post-operation, and then continuously increased until 6 months after the operation. IKQ stabilized between 6 and 12 months after surgery. IKQ measured at 60 degrees was significantly lower than that of the healthy knees 1 month after the operation; IKQ mea- sured at 180 degrees was significantly lower than that of the healthy knees from baseline to 6 months after the operation, but no significant difference was noted 12 months after the operation (data not shown).

As shown in Table 2, significant differences between the patients' two knees were observed in VITR, VLTR, VMTR, and effusion at baseline and at 1 month after the operation. The postoperative trends of these ultrasound parameters are summarized in Table 2.

Significant differences in RFD between the two knees were observed 1 month after the operation, an indication of the significant impact of the operation on RFD (Table 1). The postoperative trends in RFD were further investigated, but no significant changes were observed among the various time points. Also, no significant difference was observed between the two knees from 2 months post-surgery to the end of follow-up at 1 year (data not shown).

ROM of the operated knees 1 and 2 months after the operation was significantly lower than at baseline. ROM of the 
Table 3. ROM and WOMAC trends in pain, stiffness, and function

\begin{tabular}{|c|c|c|c|c|c|c|}
\hline & & $\begin{array}{l}\text { Pre-operation } \\
\qquad(\mathrm{n}=22)\end{array}$ & $\begin{array}{c}1 \text { month } \\
\text { post-operation } \\
(\mathrm{n}=20)\end{array}$ & $\begin{array}{c}2 \text { months } \\
\text { post-operation } \\
(\mathrm{n}=18)\end{array}$ & $\begin{array}{c}6 \text { months } \\
\text { post-operation } \\
(\mathrm{n}=16)\end{array}$ & $\begin{array}{c}12 \text { month s } \\
\text { post-operation } \\
(\mathrm{n}=11)\end{array}$ \\
\hline \multirow[t]{2}{*}{ ROM } & Operated & $103.3 \pm 14.99$ & $86.9 \pm 15.3 * 9$ & $92.8 \pm 14.4^{*}+\pi$ & $105.5 \pm 16.4 \uparrow+9$ & $105.7 \pm 10.1+t$ \\
\hline & Healthy & $120.3 \pm 10.7$ & $115.1 \pm 13.3$ & $113.3 \pm 10.1$ & $116.2 \pm 12.6$ & $112.0 \pm 13.0$ \\
\hline \multicolumn{7}{|l|}{ WOMAC } \\
\hline Pain & & $3.315 \pm 2.357$ & $2.407 \pm 1.291$ & $1.920 \pm 1.395$ & $0.899 \pm 0.710 *+\$$ & $1.414 \pm 0.968 \dagger$ \\
\hline Stiffness & & $3.568 \pm 2.116$ & $3.172 \pm 2.052$ & $2.457 \pm 1.640$ & $2.205 \pm 2.113$ & $2.479 \pm 1.491$ \\
\hline Function & & $3.321 \pm 1.837$ & $3.566 \pm 1.318$ & $1.953 \pm 1.027^{* \dagger}$ & $1.301 \pm 0.796 * \dagger$ & $1.279 \pm 0.751^{*+\dagger}$ \\
\hline
\end{tabular}

* Significant difference from baseline; $\dagger$ significant difference from 1 month after the operation; and $\ddagger$ significant difference from 2 months after the operation. I significant difference between the operated and healthy knees.

operated knees at 6 and 12 months after the operation was significantly higher than that at 1 and 2 months after the operation. However, no significant change in ROM of the healthy knee was observed. ROM of the operated knees was significantly lower than that of the healthy knees from baseline to 6 months after the operation, and was comparable to the level of the healthy knees 12 months after the operation (Table 3).

WOMAC scores for pain, stiffness, and function were used to evaluate the severity of degenerative arthritis. The mean pain score continuously decreased from baseline to 6 months after the operation, but then slightly increased 12 months after the operation. The WOMAC pain score at 6 months post-operation was significantly lower than at baseline, and at 1 and 2 months after the operation. The stiffness score decreased from baseline to 2 months after the operation, and then stabilized to the end of follow-up; no significant differences were noted among the time points. The functional score was comparable between baseline and 1 month after the operation, and continuously decreased from 1 month to 12 months after the operation. The functional scores at 2, 6, and 12 months after the operation were significantly lower than baseline and 1 month post-operation. Finally, the functional score 12 months after the operation was significantly lower than that recorded 2 months postoperation (Table 3).

\section{DISCUSSION}

The results of our study show that while strength, ROM and RFD decreased 1 month after surgery, these parameters subsequently improved over time and there were no significant differences between the operated and healthy knees 12 months after surgery. At baseline, ultrasound measurements showed significant differences in VITR, VLTR and VMTR between the operated and healthy knees, and greater effusion in the operated knees compared to the healthy knees. However, no significant difference in ultrasound measurements between the operated and healthy knees was seen at 12 months after surgery. The mean WOMAC scores for pain, stiffness and function all showed decreasing trends, and were all lower compared to the baseline at 12 months post-surgery; however, only the functional score difference was significant.
Helping patients recover strength and mobility is a major goal of minimally invasive TKA. Numerous studies have evaluated the importance of strengthening the quadriceps muscle after TKA. In addition to maximal strength, quadriceps RFD in the first 100 to $200 \mathrm{msec}$ from onset of contraction provides an alternative functional outcome measure for persons receiving $\mathrm{TKA}^{7-9}$ ).

A few other studies have traced functional recovery after minimally invasive TKA. For example, in their study of 40 subjects who underwent unilateral TKA followed by rehabilitation, including 6 weeks of outpatient physical therapy, Mizner et al. ${ }^{10)}$ found that the subjects experienced significant worsening of knee ROM, quadriceps strength, and performance in functional tests 1 month after surgery. As with our patients, in the study of Mizner et al., all functional measures had significantly improved by 6 months after surgery; quadriceps strength was the most affected ${ }^{10)}$. Other researchers have pointed to the importance of hamstring strength after surgery as a marker of return of function. Stevens-Lapsley et al. prospectively followed 30 patients undergoing TKA at 2 weeks postoperatively, and 1,3 , and 6 months after surgery, and compared them with a cross-sectional cohort of 15 healthy older adults ${ }^{11)}$. While there were no differences in strength loss or recovery between the quadriceps and hamstring muscles of the operated legs, there were differences between the untreated legs and healthy control legs. Hamstrings muscle coactivation in the operated leg during maximal quadriceps effort was elevated at 1 month (144.5\%) compared to the untreated leg. The authors suggested that quadriceps and hamstring muscle strengthening should be the focus of future rehabilitation programs. Since quadriceps strength is so important, as shown in previous studies, the subvastus approach may theoretically add more benefits to minimally invasive TKA, especially during early rehabilitation and recovery of muscle strength.

Isokinetic muscle strength testing before and after minimally invasive TKA provides helpful information on muscle strength after surgery ${ }^{7,8)}$. Schroer and colleagues studied 50 patients who underwent isokinetic muscle strength testing before surgery, and at 6 weeks, 3 months, and 1 year after unilateral TKA ${ }^{12}$. When they re-evaluated the patients 1 year after surgery, the quadriceps strength of the operated knee was equivalent to that of the untreated knee. 
This finding is similar to our results of isokinetic muscle strength, which showed no significant difference between the operated and healthy knees by 12 months after surgery. Even when the entire study population of Schroer et al. was categorized by age, weight, gender, and the presence of osteoarthritis in the untreated knee, all had equivalent strength in the quadriceps muscles of the operated and untreated sides. Rossi and colleagues also found isokinetic testing to be useful for documenting torque production before and in the early states after unilateral TKA ${ }^{13)}$. At 1 month after surgery, there was a "dip" in both knee extensor and flexor torque production. One year after surgery, the same authors found knee extensor and flexor strength asymmetry between the operated knees and unoperated contralateral knees.

Minns et al. ${ }^{14)}$ performed a systematic review and metaanalysis of studies related to physical therapy and exercise intervention for patients receiving artificial joint replacement surgery for knee arthritis. They found that in most studies intervention began 10 days to 2 months after surgery, and continued for 3 to 12 weeks. If physical therapy was the only intervention after surgery, there were smallto-moderate effects on joint flexibility and subjective improvement in function and quality of life 3 to 4 months after surgery. In comparison, exercise protocols involving functional movements were more effective than traditional exercise and training.

Generally, reducing swelling and pain, and increasing joint flexibility are initial rehabilitation goals after surgery for patients with orthopedic disorders. At the mid-stage, after joint flexibility increases, training to increase muscular strength begins. However, for the population of this study, this approach may have required more time for the muscular strength of the operated lower limb to reach presurgery status or to show no significant difference from the healthy knee. In another study, significant improvements in maximum isometric contraction within or outside the training angle and muscular power were observed even with restricted knee ROM, when isokinetic concentric and eccentric contractions involving lower angular velocity were utilized (30 degrees/sec and 90 degrees/sec) $)^{15}$. Patients who receive minimally invasive arthroplasty have restricted joint flexibility in the initial stages after surgery. However, if early training involving muscular strength and joint flexibility can be performed, a better prognosis is expected.

Many factors influence the outcome of TKA, including patient selection, implant design, and surgical technique, as well as rehabilitation programs. In 2003, 402,000 primary TKAs and 32,000 revision TKAs were performed in the US, and this number is expected to double by $2015^{16}$. Understanding how patients recover and developing better ways to follow their recovery can only help them return more quickly to normal activities. Our biomechanical strength and sonographic measurements demonstrated quadriceps recovery after minimally invasive TKA with the subvastus approach, indicating that we could utilize this pattern to better design an optimal rehabilitation protocol to the benefit of patients after TKA.

Our study had two limitations. First, the small sample size might have influenced correlations of function scores (ROM and WOMAC), and second, the condition of the operated knee might have affected the opposite knee. The latter possibility is a topic for future study.

The minimally invasive subvastus approach is superior to other TKA approaches because of its reduced surgical invasiveness and the reduction of trauma/injury to the quadriceps, which allows an accelerated rehabilitation protocol and a more favorable outcome.

\section{ACKNOWLEDGEMENT}

We thank Far Eastern Memorial Hospital and Oriental Institute of Technology for its financial support (97-5-02-514).

\section{REFERENCES}

1) Felson D: The epidemiology of osteoarthritis: prevalence and risk factors In: Osteoarthritic disorders. Rosemont: The American Academy of Orthopaedic Surgeons, 1995, pp 13-24.

2) Pramer A, Fumer S, Rice DP: Musculoskeletal conditions in the United States. Rosemont: American Academy of Orthopaedic Surgeons, 1999.

3) Blazevich AJ, Horne S, Cannavan D, et al.: Effect of contraction mode of slow-speed resistance training on the maximum rate of force development in the human quadriceps. Muscle Nerve, 2008, 38: 1133-1146. [Medline] [CrossRef]

4) Severson EP, Pagnano MW: Mini-incision total knee arthroplasty: new data. In: Orthopaedic Knowledge Update: Hip and Knee Reconstruction 4; The American Academy of Orthopaedic Surgeons 2011, pp 139-149.

5) Suetta C, Aagaard P, Magnusson SP, et al.: Muscle size, neuromuscular activation, and rapid force characteristics in elderly men and women: effects of unilateral long-term disuse due to hip osteoarthritis. J Appl Physiol, 2007, 102: 942-948. [Medline] [CrossRef]

6) Xie F, Li SC, Goeree R, et al.: Validation of Chinese Western Ontario and McMaster Universities Osteoarthritis Index (WOMAC) in patients scheduled for total knee replacement. Qual Life Res, 2008, 17: 595-601. [Medline] [CrossRef]

7) Chang $\mathrm{CH}$, Chen $\mathrm{KH}$, Yang RS, et al.: Muscle torques in total knee arthroplasty with subvastus and parapatellar approaches. Clin Orthop Relat Res, 2002, 398: 189-195. [Medline] [CrossRef]

8) Chang CH, Yang RS, Chen KH, et al.: Muscle torque in total knee arthroplasty: comparison of subvastus and midvastus approaches. Knee Surg Sports Traumatol Arthrosc, 2010, 18: 934-938. [Medline] [CrossRef]

9) Maffiuletti NA, Bizzini M, Widler K, et al.: Asymmetry in quadriceps rate of force development as a functional outcome measure in TKA. Clin Orthop Relat Res, 2010, 468: 191-198. [Medline] [CrossRef]

10) Mizner RL, Petterson SC, Snyder-Mackler L: Quadriceps strength and the time course of functional recovery after total knee arthroplasty. J Orthop Sports Phys Ther, 2005, 35: 424-436. [Medline]

11) Stevens-Lapsley JE, Balter JE, Kohrt WM, et al.: Quadriceps and hamstrings muscle dysfunction after total knee arthroplasty. Clin Orthop Relat Res, 2010, 468: 2460-2468. [Medline] [CrossRef]

12) Schroer WC, Diesfield PJ, Reedy ME, et al.: Isokinetic strength testing of minimally invasive total knee arthroplasty recovery. J Arthroplasty, 2010, 25: 274-279. [Medline] [CrossRef]

13) Rossi MD, Brown LE, Whitehurst M: Knee extensor and flexor torque characteristics before and after unilateral total knee arthroplasty. Am J Phys Med Rehabil, 2006, 85: 737-746. [Medline] [CrossRef]

14) Lowe CJ, Barker KL, Dewey M, et al.: Effectiveness of physiotherapy exercise after knee arthroplasty for osteoarthritis: systematic review and meta-analysis of randomized controlled trials. BMJ, 2007, 335: 812. [Medline] [CrossRef]

15) Barak Y, Ayalon M, Dvir Z: Transferability of strength gains from limited to full range of motion. Med Sci Sports Exerc, 2004, 36: 1413-1420. [Medline] [CrossRef]

16) Liang YC, Qiu YF, Lin KJ, et al.: Effects of open and closed-kinetic-chain exercises in patients following total knee arthroplasty. Formos J Med, 2007, 11: 130-139 (in Chinese) 